\title{
Amplification of femtosecond pulses in a passive, all-fiber soliton source
}

\author{
D. J. Richardson \\ Optoelectronics Research Centre, Southampton University, Southampton SO9 5NH, UK \\ V. V. Afanasjev and A. B. Grudinin \\ General Physics Institute, Russian Academy of Sciences, 38 Vavilov Street, Moscow 117942, Russia \\ D. N. Payne \\ Optoelectronics Research Centre, Southampton University, Southampton SO9 5NH, UK
}

Received May 1, 1992

\begin{abstract}
We report a theoretical and experimental study of the amplification of femtosecond soliton pulses generated by a passively mode-locked fiber laser in a high-gain erbium-doped fiber amplifier (EDFA). Results on the spectral and temporal evolution of 450 -fs soliton pulses propagating within an EDFA are presented. Power gains as high as $30 \mathrm{~dB}$ and the generation of sub-100-fs Raman colored solitons are observed. The results are found to be well described by the theoretical model presented.
\end{abstract}

Advances in erbium-doped amplifiers (EDFA's) and, more recently, in the generation and amplification of ultrashort pulses ${ }^{1,2}$ make the development of sub$100-$ fs passive pulse sources based on erbium-doped fiber an attractive proposition. Recently, a source of transform-limited $300-\mathrm{fs}$ pulses from a passively mode-locked fiber laser has been reported. ${ }^{3-5}$ Despite the broad gain bandwidth of erbium ions in silica $(\approx 35 \mathrm{~nm})$, the minimum pulse durations obtained so far are near $300 \mathrm{fs}$ and have an optical bandwidth that utilizes only $9 \mathrm{~nm}$ of the $35 \mathrm{~nm}$ available. The process that limits the pulse duration to this value is thought to be the soliton self-frequency shift ${ }^{6}$ (SSFS). One possible way to obtain shorter pulses is to exploit the pulse compression effects that occur during soliton amplification in a fiber amplifier. ${ }^{2}$ A passive, all-fiber circuit capable of generating ultrashort femtosecond pulses comprising a figure-eight laser ${ }^{7}$ as a master oscillator coupled to an external EDFA has recently been reported. ${ }^{8,9}$ Pulses with durations as short as 30 fs have been generated by using such a scheme. ${ }^{9}$ The source is potentially diode pumpable and provides a convenient source of ultrashort solotonic pulses. In this Letter we report the results of an experimental and a theoretical study of both the temporal and spectral characteristics of femtosecond pulses that are amplified within an EDFA external to the figure-eight laser source.

The basic experimental configuration is shown in Fig. 1 (see Ref. 9 for a detailed description of the laser). The laser produced stable trains of bandwidth-limited soliton pulses with a duration of $450 \mathrm{fs}$. The passive fiber within the circuit had the following characteristics: N.A. = $0.15, \lambda_{\text {co }}=960 \mathrm{~nm}, D=3.5 \mathrm{ps} /(\mathrm{nm} \mathrm{km})$, and $A_{\text {eff }}=$ $30 \mu \mathrm{m}^{2}$. The external amplifier (Fig. 1) consisted initially of $5.5 \mathrm{~m}$ of 800 parts in $10^{6}$ erbium-doped germanosilicate fiber $\left[\right.$ N.A. $=0.15, \lambda_{\mathrm{co}}=1230 \mathrm{~nm}$,
$D=13 \mathrm{ps} /(\mathrm{nm} \mathrm{km})$, and effective mode area $A_{\text {eff }}=$ $50 \mu \mathrm{m}^{2}$ ]. A discrete change therefore exists in both fiber dispersion and mode area between the passive fiber and the external EDFA. Owing to these fiber parameter differences and the $30 \%$ output coupling from the laser (which supports fundamental solitons intracavity ${ }^{10}$ ), the pulses at the input to the external EDFA have an energy much lower than that required for a fundamental soliton in the amplifying fiber.

In order to investigate the temporal and spectral evolution along the amplifier, the EDFA was cut back in sections of $12 \mathrm{~cm}$ from the initial starting length. In addition, the average output signal power and 980nm pump power were measured to permit the gain distribution along the amplifier to be determined. These measurements were made for two different EDFA pump powers, 320 and $410 \mathrm{~mW}$, with the input signal power remaining fixed at $80 \mu \mathrm{W}$.

Figure 2 shows the total amplifier gain (as determined from the average output power) as a function of EDFA length at a pump power of $320 \mathrm{~mW}$. A corresponding plot of the pulse duration (as determined by measuring the half-width of the background-free autocorrelation trace or, in the case of more complicated

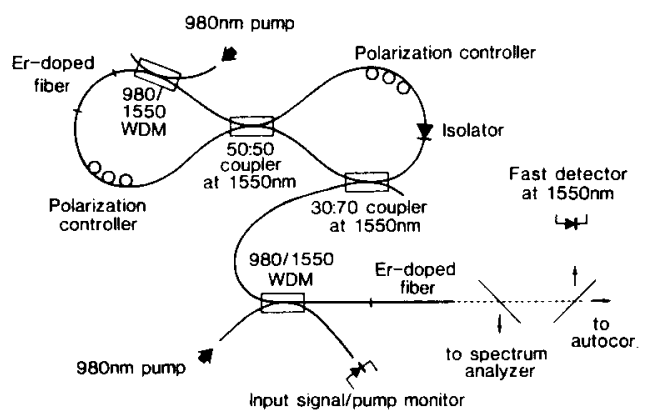

Fig. 1. Experimental configuration. WDM, wavelengthdivision multiplexer. 


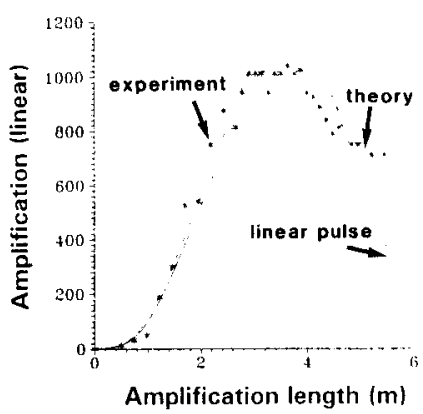

Fig. 2. Experimentally determined total power gain as a function of amplifier length for a fixed input signal power of $80 \mu \mathrm{W}$ and a fixed pump power of $320 \mathrm{~mW}$. The solid curve represents the gain model used in the computer simulation.

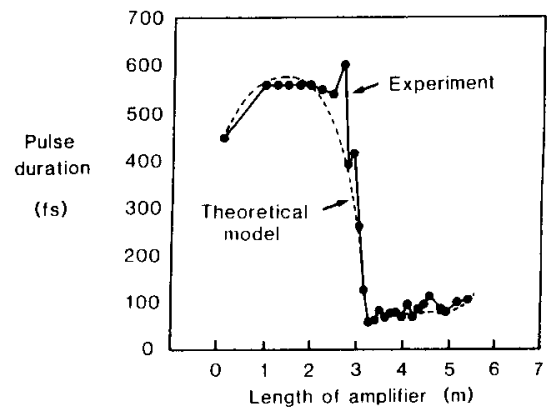

Fig. 3. Experimentally determined pulse width as a function of amplifier length for a fixed input signal power and a fixed pump power of $320 \mathrm{~mW}$. The solid curve is a best-fit result using the computer model described in the text.

autocorrelations, as determined by the half-width of the central autocorrelation feature) against amplifier length is shown in Fig. 3. Superimposed on the experimental data are the results of a theoretical simulation of the system (see below).

The evolution of the pulse along the amplifier is as follows. As explained above, owing to the discrete change of fiber parameters between the amplifier and laser fibers, the pulses input to the amplifier have an energy well below that of a fundamental soliton $(N \approx 0.2-0.3)$ and can almost be considered to propagate in the linear regime. After a short length of exponential amplification (at the smallsignal gain) the average signal power $P_{s}$ grows to that of the EDFA saturation power $P_{s}^{\text {sat }}=6 \mathrm{~mW}$, and further amplification must occur in the saturated regime. Referring to Fig. 3 , the pulse duration gradually increases owing to dispersion and the bandwidth-limited gain. After propagating a short distance within the amplifier the pulse attains an energy close to that of the fundamental soliton. Any further increase in pulse energy brings the onset of multisoliton compression effects until, at a certain pulse energy (which corresponds to a higher-order soliton $N \approx 9$ ), multisoliton compression dominates and an abrupt decrease in pulse width occurs. ${ }^{11}$ At this point, it is seen that the pulse duration contracts by a factor of 10 within a distance of only $20 \mathrm{~cm}$. In fact, it is observed that a sharp $50-\mathrm{fs}$ peak forms on a broad pedestal. Since the peak-pedestal bound energy equals zero, any perturbation (Raman term, high-order dispersion, etc.) results in temporal and spectral separation of the peak and pedestal. Moreover, the SSFS effect is $\approx 10^{4}$ times stronger for the peak than for the pedestal, and therefore SSFS acts to pull this pulse apart into individual, fundamental Raman solitons. ${ }^{6,11}$ In our experiment the local amplifier gain falls to zero at the approximate point of pulse-width collapse, as evidenced by the peak amplification, which occurs at $\approx 3.2 \mathrm{~m}$ of EDFA length (see Fig. 2). Numerical simulations show that this is merely a coincidence and that, in general, multisoliton collapse can occur at any point within the amplifier provided that the pulse has gained sufficient energy. The individual Raman solitons shift their central frequency as they propagate and thus rapidly move out of the $\mathrm{Er}^{3+}$ absorption band of the amplifier fiber. They then pass with minimal attenuation through the remaining (noninverted) section of the doped fiber. Eventually the effects of third-order dispersion lead to an increase in the pulse width of the Raman soliton to $\approx 90 \mathrm{fs}$, and the velocity of the frequency shift $\mathrm{d} \Omega / \mathrm{d} z \propto \tau_{0}^{-4}$ falls strongly.

Note that after the point of pulse-width collapse the Raman soliton and its pedestal become well separated in time. They are, however, not fully spectrally separated, and superposition of the two components can result in complex spectra. ${ }^{9}$ A background-free autocorrelation trace and spectrum of a 90 -fs soliton pulse obtained at the end of a 6-m length of amplifier fiber is shown in Fig. 4. Note the smooth sech ${ }^{2}$-like soliton spectrum that is distorted by the spectral component of the nonsoliton pedestal at the initial signal wavelength. Fortunately, the pedestal and other residual light at the input signal wavelength lie within the $\mathrm{Er}^{3+}$ absorption band and can be filtered out by using a further (unpumped) length of amplifier fiber.

A numerical simulation was undertaken based on modeling the propagation of the pulse within the amplifier. The following nonlinear Schrödinger equation was employed, which incorporates terms to describe the amplification process (including the effects of amplifier saturation and pump depletion along the amplifier length), resonant dispersion, stimulated Raman scattering, and third-order

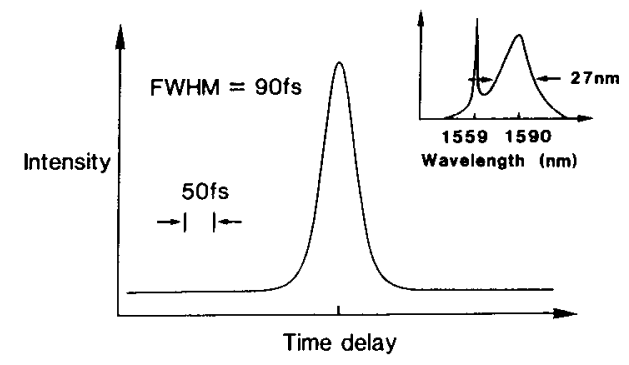

Fig. 4. Background-free autocorrelation trace and optical spectrum of 90-fs Raman solitons obtained at the amplifier output for a pump power of $200 \mathrm{~mW}$ and an amplifier length of $5 \mathrm{~m}$. The spike at the input signal wavelength is due to incomplete energy transfer from the amplified input signal to the Raman soliton. 
dispersion ${ }^{12}$ :

$$
\begin{aligned}
i \frac{\partial \psi}{\partial z}= & \frac{1}{2} \frac{\partial^{2} \psi}{\partial \tau^{2}}+i \beta_{3} \frac{\partial^{3} \psi}{\partial \tau^{3}} \\
& +(1-\beta)|\psi|^{2} \psi+\beta Q \psi+\frac{G(z)}{2} P,
\end{aligned}
$$

where the dimensionless complex amplitude is normalized to the amplitude of a fundamental soliton pulse of duration $\tau_{0}: \quad|\psi|^{2}=\left[8 \pi \beta_{2} /\left(\tau_{0}{ }^{2} k n_{2} c n_{0}\right)\right]^{1 / 2}$. The distance $z$ is expressed in dispersion lengths $L_{d}=\tau_{0}{ }^{2} / \beta_{2}$, and the running time $\tau=(t-z / u)$ is normalized to $\tau_{0}$, where $u$ is the group velocity of the pulse. The parameter $\beta \approx 0.2$ determines the Raman contribution to the nonlinear refractive index. The amplification parameter $G(z)=G_{0} g(z)$, where $G_{0}=L_{d} / L_{a}, L_{\alpha}$ is the (1/e) amplification length (for undepleted pump) and $g(z)$ is described below.

The amplitude of the molecular oscillations $Q$ as used in the semiclassical treatment of stimulated Raman scattering is given by

$$
\mu^{2} \frac{\partial^{2} Q}{\partial t^{2}}+2 \mu \delta \frac{\partial Q}{\partial t}+Q=|\psi|^{2}
$$

in which $\mu=\left(\tau_{0} \Omega_{R}\right)^{-1}, \delta=\left(T_{2}{ }^{R} \Omega_{R}\right)^{-1}$, and the real amplitude $Q$ is normalized to $Q_{n}=\alpha_{Q}\left|\psi_{s}\right|^{2} /\left(4 M \Omega_{R}{ }^{2}\right)$, where $M$ is the effective molecular mass, $\Omega_{R}=8.3 \times$ $10^{13} \mathrm{~s}^{-1}$ is the resonance frequency, $T_{2}{ }^{R}=75 \mathrm{fs}$ is the relaxation time associated with the spontaneous Raman scattering linewidth, and $\alpha_{Q}$ is the electron polarizability of the molecule.

The dimensionless complex amplitude of the active ion polarization $P$ satisfies the following equation:

$$
\gamma_{a} \frac{\partial P}{\partial \tau}+P\left(1+i \gamma_{a} \Delta \Omega\right)=i \psi
$$

which describes Lorenzian gain of bandwidth $\gamma_{a}{ }^{-1}=$ $\tau_{0} / T_{2} . \quad T_{2} \approx 80 \mathrm{fs}$ is the relaxation time associated with the amplification bandwidth $(\approx 35 \mathrm{~nm})$, and $\Delta \Omega=\tau_{0}\left(\omega-\omega_{12}\right)$, where $\omega_{12}$ is the resonant frequency. We incorporate the $z$ dependence of the gain into Eq. (1) as $G(z)=G_{0} g(z)$. From the rate equations for a three-level system one can derive the approximate expression for amplifier gain as a function of pump power $P_{p}(z)$ and signal power $P_{s}(z)$ along the amplifier length as

$$
g(z)=\left[\frac{P_{p}(z)}{P_{p}^{\text {th }}}-1\right]\left[\frac{P_{p}(z)}{P_{p}^{\text {th }}}+1+\frac{2 \epsilon(z) P_{s}(z)}{\epsilon(0) P_{s}^{\text {sat }}}\right]^{-1},
$$

where

$\epsilon(z)$

$$
=\int_{-\infty}^{\infty} \frac{1}{\left(1+\gamma_{a}^{2} \Omega^{2}\right)}|\psi(\Omega, z)|^{2} \mathrm{~d} \Omega / \int_{-\infty}^{\infty}|\psi(\Omega, z)|^{2} \mathrm{~d} \Omega
$$

and $P_{p}^{\text {th }}=3 \mathrm{~mW}$ is the pump saturation (bleaching) power. Equation (4) takes into account the $z$ dependence of the pump intensity [we use experimental data for $P_{p}(z)$ ] and gain saturation by the signal. Equation (5) shows that only spectral components within the amplification band contribute to saturation of the amplifier. We assume that the input pulse has a $\operatorname{sech}^{2} \tau$ time envelope with 450 -fs duration. The experimental curves were fitted by the appropriate choice of parameter $G_{0}$.

As can be seen (Figs. 2 and 3), the model is able to predict well the general features of the system behavior. At longer fiber lengths, or higher amplifier gain, the model predicts more than one colored soliton generation after the collapse, as observed experimentally. ${ }^{9}$ The model further indicates that by judicious choice of laser output coupling and suitable matching of the amplifier and laser fiber dispersion and mode size, further pulse-width reduction should be possible at significantly lower amplifier powers, which would make the unit a potentially diode-pumpable source of sub-100-fs soliton pulses.

In conclusion, we have presented experimental and theoretical results on the amplification of femtosecond soliton pulses. The computer simulation is found to describe the experimental data well. Single-stage compression of pulses from 450 to 50 fs has been obtained. The generation of single or multiple Raman solitons has been observed starting from an almost linear pulse.

\section{References}

1. I. Y. Krushchev, A. B. Grudinin, E. M. Dianov, D. V. Korobkin, V. A. Semenov, and A. M. Prokhorov, Electron. Lett. 26, 456 (1990).

2. K. Kurokawa and M. Nakazawa, Appl. Phys. Lett. 58, 2871 (1991).

3. I. N. Duling III, Electron. Lett. 27, 544 (1991).

4. D. J. Richardson, R. I. Laming, D. N. Payne, V. Matsas, and M. W. Phillips, Electron. Lett. 27, 542 (1991).

5. M. Nakazawa, E. Yoshida, and Y. Kimura, Appl. Phys. Lett. 59, 2073 (1991).

6. V. V. Afanasyev, V. V. Vyloukh, and V. N. Serkin, Opt. Lett. 15, 489 (1990).

7. A. G. Bulushev, E. M. Dianov, and O. G. Okhotnikov, Opt. Lett. 16, 88 (1991).

8. D. J. Richardson, A. B. Grudinin, and D. N. Payne, Electron. Lett. 28, 778 (1992).

9. D. J. Richardson, A. B. Grudinin, and D. N. Payne, in Digest of Conference on Nonlinear Guided-Wave Phenomena (Optical Society of America, Washington, D.C., 1992), PDP8.

10. A. B. Grudinin, D. J. Richardson, and D. N. Payne, Electron. Lett. 28, 67 (1992).

11. G. P. Agrawal, Phys. Rev. A 44, 7493, (1991). See also D. J. Richardson, V. V. Afanasjev, A. B. Grudinin, and D. N. Payne, in Technical Digest of Eighth International Conference on Ultrafast Phenomena (Ecole Polytechnique/ENSTA, Palaiseau, France, 1992), paper ME4.

12. V. V. Afanasjev, E. M. Dianov, A. M. Prokhorov, and V. N. Serkin, Pisma Zh. Tek. Fiz. 16, 67 (1990). 